Valentina Corda, Giovanni Monni*, Federica Murgia, Alba Piras, Rosa Maria Ibba, Frank A. Chervenak and Laurence B. McCullough

\title{
Induced abortion and COVID-19 as contributing factors to declining fertility in Sardinia
}

https://doi.org/10.1515/jpm-2021-0289

Received June 11, 2021; accepted July 22, 2021;

published online August 12, 2021

\section{Abstract}

Objectives: Decreasing fertility implies considerable public health, societal, political, and international consequences. Induced abortion (IA) and the recent COVID-19 pandemic can be contributing factors to it but these have not been adequately studied so far. The purpose of this paper is to explore the relation of IA incidence and the COVID-19 pandemic to declining rates of delivery, as per our Sardinian experience.

Methods: We analyzed the registered data from the official Italian statistics surveys of deliveries and IA in the last 10 years from 2011 to 2020 in Sardinia.

Results: A total of 106,557 deliveries occurred and a progressive decrease in the birth rate has been observed. A total of 18,250 IA occurred and a progressive decline has been observed here as well. The ratio between IA and deliveries remained constant over the decade. Between 2011 and 2019 a variation of $-4.32 \%$ was observed for IA while in the last year, during the COVID-19 pandemic the decrease of the procedures was equal to $-12.30 \%$. For the deliveries, a mean variation of the $-4.8 \%$ was observed between the 2011 and the 2019 while in the last year, during the COVID-19 pandemic the decrease was about $-9 \%$. Considering the about 30\% reduction of live births between 2011 and 2020, there is an almost proportional reduction in IA.

*Corresponding author: Giovanni Monni, MD, Department of Obstetrics and Gynecology, Prenatal and Preimplantation Genetic Diagnosis, Fetal Therapy, Microcitemico Pediatric Hospital “A. Cao", Via Edward Jenner SNC, 09121 Cagliari, Sardinia, Italy, Phone: +0039 070 52965546/7, Fax: +003907052965514,

E-mail: prenatalmonni@tiscali.it

Valentina Corda, Federica Murgia, Alba Piras and Rosa Maria Ibba, Department of Obstetrics and Gynecology, Prenatal and Preimplantation Genetic Diagnosis, Fetal Therapy, Microcitemico Pediatric Hospital “A. Cao”, Cagliari, Sardinia, Italy Frank A. Chervenak and Laurence B. McCullough, Department of Obstetrics and Gynecology, Zucker School of Medicine at Hofstra/ Northwell and Lenox Hill Hospital, New York, NY, USA
Conclusions: Public policy responses to decreasing fertility, especially pronatalist ones, would be provided with evidence base about trends in delivery and IA and women's decision making.

Keywords: birth rate decline; COVID-19; induced abortion; mifepristone.

\section{Introduction}

Decreasing fertility has become a global phenomenon with potentially serious and far-reaching public health, societal, political, and international consequences. Induced abortion (IA) as a contributing factor of decreasing fertility has not been adequately studied. The purpose of this paper was to use the experience in Sardinia to investigate the incidence of IA relative to declining rates of delivery, above all in COVID-19 pandemic [1]. On March 11th 2020, the World Health Organization made the assessment that the Coronavirus disease 19 (COVID-19) can be characterized as a pandemic [2]. The authors also explored how the pandemic affected the rate of IA relative to the delivery rate.

\section{Materials and methods}

In 1978, a law was passed in Italy which set forth the regulations governing the procedures for obtaining an IA, also denoted locally as "voluntary interruption of pregnancy". According to this law, all women can request an IA during the first 90 days of gestation due to health, economic, social, or familial reasons. Before undergoing an IA, the woman must obtain a pregnancy certificate from her general practitioner, or from a private physician or a public clinic.

The law also allows IA in the second trimester of pregnancy before viability as well but only when the life of the woman is at risk or if the fetus is affected by genetic or other severe malformations which would expose the mother to serious psychological or physical consequences.

In Italy, the scope of IA includes "selective feticide” and “embryo reduction in multiple pregnancies" as well [3, 4].

The IA is performed free-of-charge at either at a healthcare structure of the National Healthcare System or in a private setting authorized by regional health authorities. 
In Italy, IA can be performed surgically or via medication. Medical IA is performed using Mifepristone, also known as RU-486. It is the medication typically used in combination with Misoprostol to bring about an abortion during pregnancy. This combination is $97 \%$ effective during the first 63 days of gestation.

The marketing of Mifepristone in Italy has been authorized by the Italian Drug Agency since July 2009. The indications for its use differ than those in other countries. OMS requires hospitalization from the time of the taking of the drug until verification of the expulsion of the product of conception.

As observed in other countries and reported internationally in scientific literature, the Mifepristone-Prostaglandin combination is a highly effective method for IA in early gestation. In fact, in Italy as well, the necessity to perform hysterosuction Karman or revision of the uterine cavity to complete the IA or control the hemorrhage has occurred only in $5.3 \%$ of the cases; this percentage is in line with what has been reported in literature [5].

In itself, this method is not better than the other techniques employed but is an additional option, effective and safe, which supplements the choice range of the woman that should face an IA.

During the COVID-19 pandemic, unlike in other European countries, the IA gestational age limits as well as healthcare pathway and access to hospital services have remained unmodified. The COVID-19 pandemic determined diametrically opposite changes in IA policy around the world. Some countries, such as Hungary, sospende IA due to a ban of non-life threatening surgeries, while others finally legalized it [6].

We report the IA results in Sardinia which is an Italian island in the Mediterranean Sea with a population of 1.7 million. For this aim, the registered data of deliveries and IA in the last 10 years in Sardinia were analyzed through Graph-Pad PRISM software (version 7.01, GraphPad Software, Inc., CA, USA).

We analyzed data from 2011 to 2019 obtained from the official national statistics surveys (National Institute of Statistics-ISTAT) which detect cases of IA since 1979, after the enforcement of Law 194/78, in agreement with the Italian Ministry of Health and all Italian regions. Data are collected through a declaration form compiled by the physician who performs IA. This form provides information on the woman such as date and place of birth, current residence, citizenship, marital status, educational qualification, professional/ non-professional status, branch of economic activity; reproductive anamnesis; gestational age; weeks of amenorrhea, possible presence of malformations. The form also includes details on the termination of pregnancy: date, place and type of IA procedure; date of certification; authorization certificate; urgency; analgesic therapy; hospitalization and its duration; complications.

Since the statistics for year 2020 have not yet been reported officially by ISTAT, we obtained data from hospital records and additional sources.

\section{Results}

A total of 106,557 (range 8.071-12.650, mean=10.656, $\mathrm{SD}=1.534$ ) deliveries occurred over the 10 years from 2011 to 2020. A progressive decrease in the birth rate has been observed in Sardinia (Figure 1). In particular, the percentage of decrease was $36.2 \%$. In absolute value, the decrease was 4,579 deliveries in the considered time frame. On the other hand, a total of 18,250 (range 1.393-2.232, mean=1.825, $\mathrm{SD}=270.1$ ) IA occurred over the 10 years from 2011 to 2020 (Figure 1). Also in this case, a progressive decline has been observed in Sardinia with a decrease of $34.14 \%$. In absolute value, the decrease was 722 . The coefficients of variation for the two phenomena were similar, $13.07 \%$ for the deliveries and $14.45 \%$ for the IA (Figure 1). Moreover, the ratio between the IA and deliveries remained constant over the decade considered (about 0.17). Considering the average percentage change of the two phenomena, it is important to emphasize that between 2011 and 2019 a variation of the $-4.32 \%$ was observed for the IA while in the last year, during the COVID-19 pandemic the decrease of the procedures was equal to $-12.30 \%$. For the deliveries, a mean variation of $-4.8 \%$ was observed between 2011 and 2019 while in the last year, during the COVID-19 pandemic the decrease of the events was about $-9 \%$. All the values are reported in Table 1.

In particular, the number of births was 8,858 in 2019 and 8,071 in 2020 with a decrease rate of $-8.90 \%$. The number of IA was 1,588 in 2019 and 1,393 in 2020 with a

\section{A}

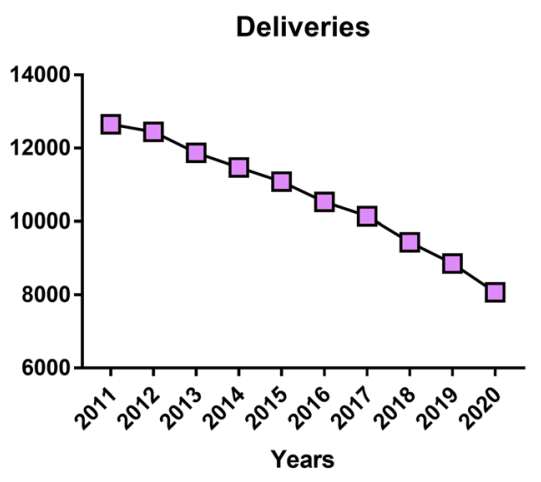

B

IA

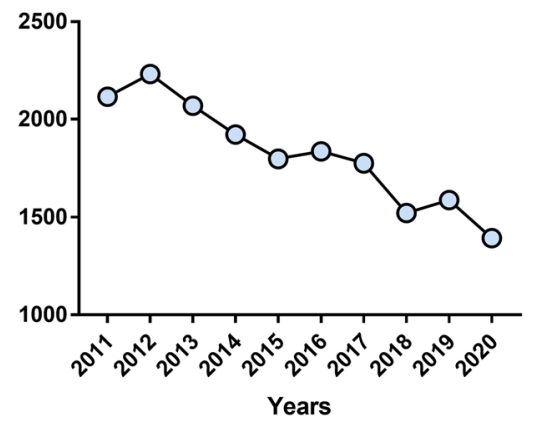

Figure 1: Decline of number of deliveries and induced abortion (IA) in Sardinia in the last decade 2010-2020. 
Table 1: Percentage variation and absolute value of deliveries and induced abortion (IA) in Sardinia from 2011 to 2020.

\begin{tabular}{lrrrrr}
\hline Years & \multicolumn{2}{c}{ Deliveries } & & \multicolumn{2}{c}{ IA } \\
\cline { 2 - 3 } \cline { 5 - 6 } & $\begin{array}{c}\text { Percentage } \\
\text { variation }\end{array}$ & $\begin{array}{r}\text { Absolute } \\
\text { value }\end{array}$ & $\begin{array}{r}\text { Percentage } \\
\text { change }\end{array}$ & $\begin{array}{r}\text { Absolute } \\
\text { value }\end{array}$ \\
\hline $2011-2012$ & $-1.60 \%$ & -206 & $5.50 \%$ & 117 \\
$2012-2013$ & $-4.60 \%$ & -572 & & $-7.30 \%$ & -163 \\
$2013-2014$ & $-3.40 \%$ & -399 & & $-7.10 \%$ & -147 \\
$2014-2015$ & $-3.40 \%$ & -391 & & $-6.50 \%$ & -124 \\
$2015-2016$ & $-5.00 \%$ & -555 & $2.10 \%$ & 38 \\
$2016-2017$ & $-3.70 \%$ & -385 & $-3.30 \%$ & -61 \\
$2017-2018$ & $-6.90 \%$ & -704 & $-14.30 \%$ & -253 \\
$2018-2019$ & $-6.10 \%$ & -580 & & $4.30 \%$ & 66 \\
$2019-2020$ & $-8.90 \%$ & -787 & $-12.30 \%$ & -195 \\
\hline
\end{tabular}

Table 2: Number of deliveries and induced abortion (IA) in Sardinia in 2019 and 2020 during COVID-19 pandemic.

\begin{tabular}{lrr}
\hline Years & Delivery, $\mathbf{n}$ & IA, $\mathbf{n}$ \\
\hline 2019 & 8,858 & 1,588 \\
2020 & 8,071 & 1,393 \\
$\Delta \%$ & -8.90 & -12.30 \\
\hline
\end{tabular}

decrease rate of $-12.30 \%$ during COVID-19 pandemic, as reported in Table 2.

The decline in fertility and the postponement of births explain only partially the reduction of IA. Considering the about 30\% reduction of live births between 2011 and 2020, there is an almost proportional reduction in IA.

\section{Discussion}

The major finding of this study is that rates of both deliveries and IA demonstrate a clear trend of reduction in occurrence that is almost proportional during the first eight years of the period studied. Both downward trends remained steady during the ninth year, which includes the COVID-19 pandemic. The percent decrease in IA was 50\% more than the decrease for deliveries for the ninth year, 2019-2020. This difference could be related to the concurrent decrease of the percentage of immigrant women arriving in Sardinia, subsequent to the several local COVID-19 lockdowns and strict travel limitations worldwide. Immigrant women generally increase the IA rate in Italy.
Little is known about the decision making of women of child-bearing potential in Sardinia about getting and remaining pregnant. Well-conducted qualitative and quantitative research would advance our understanding of women's decision-making processes. The results of such research would be ethically significant, because they would illuminate how women are exercising their autonomy-based reproductive rights about getting and remaining pregnant [7].

\section{Conclusions}

This parallel trend of decrease in deliveries and IA, if it also characterizes Italy at large, may help to understand better the clinical dimensions of one of the most important and urgent policy challenges that Italy confronts: the declining birth rate. Investigators in other countries with declining fertility should undertake similar studies along with the above described studies of women's decision making. Public policy responses to decreasing fertility, especially pronatalist policies, would then provide an evidence base that would include evidence about trends in delivery and IA and about women's decision making.

Acknowledgments: The authors of this manuscript would like to thank Fondazione di Sardegna and Boyana Petrova Tsikalova, MA in English Philology for editing assistance. Research funding: None declared.

Author contributions: All authors have accepted responsibility for the entire content of this manuscript and approved its submission.

Competing interests: Authors state no conflict of interest. Informed consent: Not applicable.

Ethical approval: Not applicable.

\section{References}

1. Monni G, Corda V, Iuculano A. Prenatal screening diagnosis and management in the era of coronavirus: the Sardinian experience. J Perinat Med 2020;48:943-9.

2. World Health Organization (WHO). WHO Director-General's opening remarks at the media briefing on Covid 19; 2020. Available from: https://www.who.int/director-general/ speeches/detail/who-director-general-s-opening-remarks-atthe-media-briefing-on-covid-19-11-march-2020.

3. Monni G, Illescas T, luculano A, Floris M, Mulas F, McCullough LB, et al. Single center experience in selective feticide in high-order 
multiple pregnancy: clinical and ethical issues. J Perinat Med 2016; 44:161-6.

4. Chervenak FA, McCullough LB. An ethically justified practical approach to offering, recommending, performing, and referring for induced abortion and feticide. Am J Obstet Gynecol 2009;201: 560.e1-6.

5. WHO Task Force on Post-ovulatory Methods of Fertility Regulation. Comparison of two doses of mifepristone in combination with misoprostol for early medical abortion: a randomised trial. BJOG 2000;107:524-30.

6. Moreau C, Shankar M, Glasier A, Cameron S, Gemzell-Danielsson K. Abortion regulation in Europe in the era of COVID-19: a spectrum of policy responses. BMJ Sex Reprod Health 2020;0:1-8.

7. McCullough LB, Coverdale JH, Chervenak FA. Professional ethics in obstetrics and gynecology. New York and Cambridge: Cambridge University Press; 2020. 